\title{
Piezo1 mechanosensitive channels: what are they and why are they important
}

\author{
Pietro Ridone $^{1,2} \cdot$ Massimo Vassalli $^{3} \cdot$ Boris Martinac ${ }^{1,4}$ (D) \\ Received: 27 July 2019 / Accepted: 27 August 2019/Published online: 7 September 2019 \\ (C) The Author(s) 2019
}

\begin{abstract}
Mechanosensitive (MS) ion channels are integral membrane proteins which play a crucial role in fast signaling during mechanosensory transduction processes in living cells. They are ubiquitous and old in the evolutionary sense, given their presence in cells from all three kingdoms of life found on Earth, including bacterial, archaeal, and eukaryotic organisms. As molecular transducers of mechanical force, MS channels are activated by mechanical stimuli exerted on cellular membranes, upon which they rapidly and efficiently convert these stimuli into electrical, osmotic, and/or chemical intracellular signals. Most of what we know about the gating mechanisms of MS channels comes from the work carried out on bacterial channels. However, recent progress resulting from identification and structural information of eukaryotic K2P-type TREK and TRAAK as well as Piezo1 and Piezo2 MS channels has greatly contributed to our understanding of the common biophysical principles underlying the gating mechanism and evolutionary origins of these fascinating membrane proteins. Using Piezol channels as an example, we briefly describe in this review what we have learned about their biophysics, physiological functions, and potential roles in "mechanopathologies."
\end{abstract}

Keywords Lipid bilayer · Force-from-lipids · Force-from-filament · Transbilayer pressure profile · Patch clamp · Liposome reconstitution

\section{Introduction}

Living cells have throughout evolution learned to cope with and respond to various mechanical stimuli by possessing a range of proteins associated with their cellular envelope, including cell wall, cytoskeleton (CSK), extracellular matrix (ECM), and membrane proteins. Among them, mechanosensitive (MS) ion channels are force-sensing integral membrane proteins, whose function is tightly linked to the lipid bilayer of cell membranes. MS channels operate on a millisecond time scale and are thus

Boris Martinac

b.martinac@victorchang.edu.au

1 Mechanosensory Biophysics Laboratory, Victor Chang Cardiac Research Institute, 405 Liverpool St, Darlinghurst, NSW 2010, Australia

2 School of Biotechnology and Biomolecular Science, University of New South Wales, Kensington, NSW 2052, Australia

3 Institute of Biophysics, National Research Council, Genoa, Italy

4 St Vincent's Clinical School, University of New South Wales, Darlinghurst, NSW 2010, Australia usually found at the very origin of cellular signaling pathways involved in mechanosensory transduction processes. Given the omnipresence of mechanical stimuli acting on the cell membrane, it is important to note that the function of integral membrane proteins other than MS channels, such as GPCRs or membrane-associated phospholipases, can also be modulated by the application of mechanical force (Martinac and Cox 2017; Storch et al. 2012; Lehtonen and Kinnunen 1995). MS ion channels, however, present an excellent example of coupling membrane proteins and their structural dynamics to the mechanics of the cell membrane. Although a large number of MS channels have over the last 30 odd years been identified at the molecular level in organisms encompassing all types of life forms from bacteria to humans, the 3D structure of only several of them has been determined by X-ray crystallography or more recently by cryo-electron microscopy (cryo-EM) (Bass et al. 2002; Chang et al. 1998; Dong et al. 2015; Brohawn et al. 2012; Ge et al. 2015; Guo and Mackinnon 2017; Zhao et al. 2018; Saotome et al. 2018; Murthy et al. 2018; Wang et al. 2019). Their function has been studied in a great variety of cells and tissues using a range of experimental and computational approaches, as described in many excellent reviews, some of 
which are listed here (Hamill and Martinac 2001; Sachs 2010; Martinac 2011; Kung 2005; Kocer 2015; Gillespie and Walker 2001; Chalfie 2009; Martinac and Cox 2017). By focusing on Piezo1 ion channels, we provide in this article a brief overview on the following: (i) what has been learned about how MS channels detect mechanical stimuli, (ii) how they convert these stimuli into structural transitions between the closed and open channel structures, and (iii) how different cellular components may be involved in these structural changes.

\section{Diversity of mechanosensitive channels and importance of Piezo1}

MS ion channels are pore-forming membrane proteins that gate in response to mechanical stimuli exerted on the cell membrane. By switching between the closed and open conformations, these channels allow ions and other solutes to flow across the cellular membranes (Martinac and Cox 2017; Hamill and Martinac 2001). They have been shown to play a key role in many physiological processes associated with mechanosensory transduction, including osmoregulation in plants, fungi, and bacteria as well as hearing, touch, proprioception, and blood flow regulation in mammalian cells (Martinac 2004; Ranade et al. 2015; HonorÉ et al. 2015). At present, MS channels are considered to be a major class of mechanosensory membrane proteins acting as molecular transducers of mechanical stimuli on a millisecond time scale and converting them into electrical and/or chemical intracellular signals bypassing millions of ions and solutes when they are open (Martinac 2013). A good example is given by members of the bacterial MscL and MscS channel families that by opening upon activation by mechanical force quickly release osmolytes from bacterial cells, which become swollen due to the increase in the turgor pressure. By responding to membrane tension resulting from the increase in turgor, MscL- and MscS-like channels protect the bacterial cells from bursting upon a hypo-osmotic shock (Martinac 2011; Hamill and Martinac 2001).

To define an MS ion channel as a truly mechanically gated channel, its activity must be controlled over the whole dynamic range from the fully closed to the fully open state by mechanical force alone (Fig. 1).

The forces that lie within the range experienced for these channels in vivo correspond to membrane tensions of up to $25 \mathrm{mN} / \mathrm{m}$, which roughly corresponds to the average lytic tension of the lipid bilayer of cellular membranes (Bavi et al. 2014; Nomura et al. 2012). It is worth noting that, besides the true MS channels, there are also ion channels that can be modulated by mechanical stimuli but their activity is primarily dependent on factors other than mechanical force such as membrane potential or ligand concentration (Martinac and Cox 2017).

Given that a large proportion of our knowledge on MS channels comes from "non-specialized" cells, the necessary and sufficient criteria that have generally been accepted as a proof of the true physiologically relevant mechanosensitivity of an ion channel are as follows:

1. The ion channel is required for cellular mechanotransduction and its removal abolishes the mechanosensory response.

2. Structural mutations introduced into the channel alter its functional properties and consequently, they affect the mechanosensory cellular response.

3. Heterologous expression of the channel gives rise to robust mechanosensory responses.

It is, nevertheless, important to remember that although very useful for experimental characterization of MS channels, these basic criteria cannot account for the biological complexities arising from the structural plasticity of many ion channel

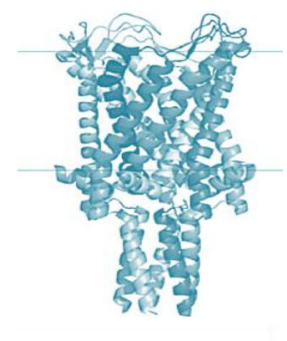

MscL

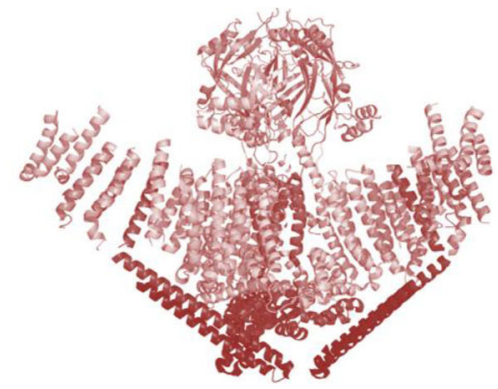

Piezo1

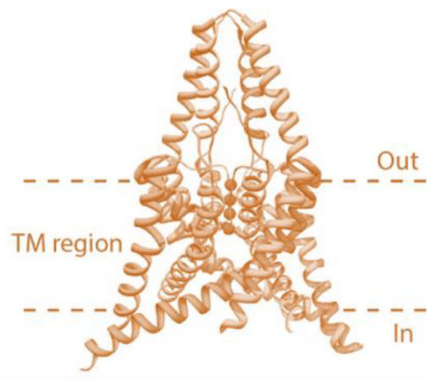

TREK-2
Fig. 1 Examples of truly mechanically activated ion channels. MscL, Piezo1, and TREK-2 are activated and controlled by mechanical force over their whole dynamic range from the fully closed to the fully open state. The 3D X-ray structure of MscL from Mycobacterium tuberculosis shows the channel homopentamer in the closed state (adapted from Chang et al. (1998)). The Piezo1 structure determined by cryo-EM shows the first low-resolution structure of the trimeric channel in its closed conformation (adopted from Ge et al. (2015)). The higher resolution structure has later been determined by several laboratories (Guo 2017;
Zhao et al. 2018; Saotome et al. 2018). Crystal structure of the human TREK-2 channel, a member of the K2P family which forms dimers. Each monomer adds two-pore loops to the structure to end up with pseudotetrameric assembly (Dong et al. 2015). One of the structural characteristics of the truly mechanically gated channels is recently resolved lipidbinding domains, similar to the MscL N-terminus (Bavi et al. 2016). Such lipid-binding structural domains were identified in the MscS-like channels, K2P family of channels (TREK/TRAAK), and most recently Piezo1 ion channels (Bavi et al. 2017a, b) 
families and functional redundancy characteristic of many physiological systems. This can make it difficult to unequivocally determine the molecular origins of mechanosensitive currents encountered in a large variety of living cells. The role in physiology/ical processes of many MS channels is beyond doubt as attested by the example of Piezo1 channel, which this review is focusing on. Piezo1, a member of the newly discovered family of MS channels (Coste et al. 2010), is a truly mechanosensitive channel as will be shown and discussed later in this review. Together with Piezo2, it is one of the long-sought principal types of molecular force sensors in mammalian cells. It enables cells to decode various physical stimuli and presents an essential component of many mechanosensory processes, including vascular development and erythrocyte volume homeostasis in humans. Despite only recently being identified, Piezol gene variants have been linked to several human pathologies such as hereditary xerocytosis (Fotiou et al. 2015) and generalized lymphatic dysplasia (Lukacs et al. 2015). Thus, the medical importance of Piezo1, as well as other MS channels, has been recognized given the role that abnormal MS channel activity plays in the pathophysiology of many diseases. These diseases are collectively referred to as mechanochannelopathies, which will be dealt in more detail later in this review.

\section{A quest for the general mechanism of gating mechanosensitive channels by mechanical force}

The exact mechanism(s) of MS channel gating are currently largely unknown due to the relatively young age of this research area in mechano-biology/physiology. Despite the inherent complexities in mechanical force transduction in mammalian systems, there are two main gating paradigms that have generally been accepted to apply to gating of MS channels in both prokaryotic and eukaryotic cells. One of the paradigms is known as the force-from-lipids (Martinac et al. 1990; Kung 2005; Teng et al. 2015) and the other one as the force-from-filament (Fig. 2) (Chalfie 2009; Katta et al. 2015).

\section{Force-from-lipids or force-from-filament?}

The force-from-lipids (FFL) gating paradigm implies that mechanical force activates MS channels through the lipid bilayer alone with no requirement for other cellular components. It has been proposed about 30 years ago in conjunction with studies on bacterial MS channels (Martinac et al. 1990). Unlike bacterial cells, animal and human cells do not have the rigid cell wall, but they possess instead membrane invaginations in the form of ruffles, folds, and microvilli. These invaginations contribute excess membrane area, which by unfolding upon stress protect the cell membrane from excessive strain. In addition, the membrane of mammalian cells is supported by ECM as external scaffolding, whereas CSK is strengthening the membrane from the intracellular side.

For most of the time, it was believed that only prokaryotic (bacterial and archaeal) MS channels were gated according to the FFL paradigm because bacteria have only a rudimentary CSK, from which modern mammalian CSK might have evolved (Barry and Gitai 2011), and rely largely on the cell wall to protect their fragile cytoplasmic membrane. Importantly, it has recently been shown that mammalian MS channels, including K2P-type TREK-1, TREK-2, and TRAAK (Berrier et al. 2013; Brohawn et al. 2014b; Brohawn et al. 2014a) ion channels as well as Piezo1 (Cox et al. 2016a; Cox et al. 2016b; Syeda et al. 2016) and OSCA channels (Murthy et al. 2018), are also gated by the FFL mechanism.

\section{Transbilayer pressure profile and gating of MS channels}

The distinction between the FFL and force-from-filaments (FFF) mechanism of gating MS channels by mechanical force seems to be a false dichotomy, which is confusing rather than clarifying the molecular principles. Thus, the question about a general mechanism that may reduce the two paradigms to a single basic principle of MS channel mechanosensitivity remains to be addressed. As a possibility, the transbilayer pressure profile (Fig. 3) appears as an ideal candidate for reducing the two gating mechanisms to the FFL paradigm only.
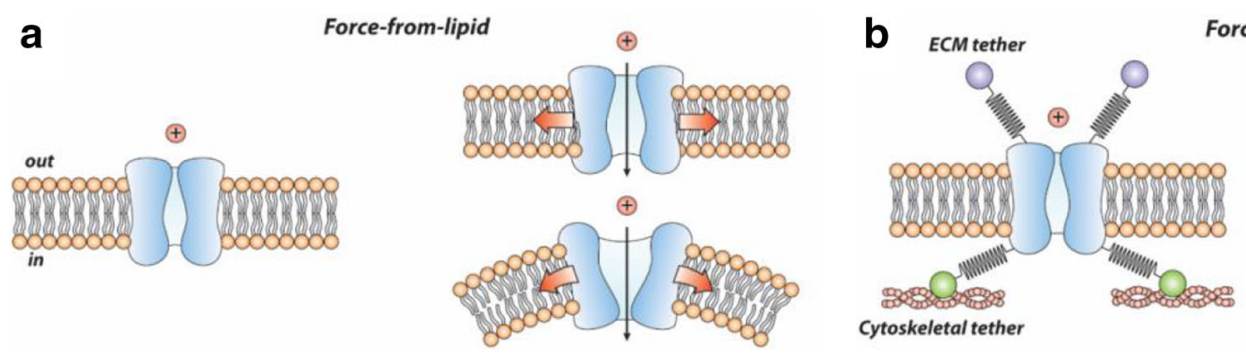

Force-from-filament

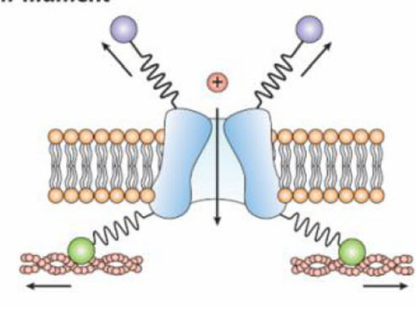

Fig. 2 Gating paradigms of MS channels. The two main generally accepted gating paradigms of MS channels are defined according to the cell membrane components (lipid bilayer (a) or ECM/CSK (b)) transmitting the activation force directly to MS channels 


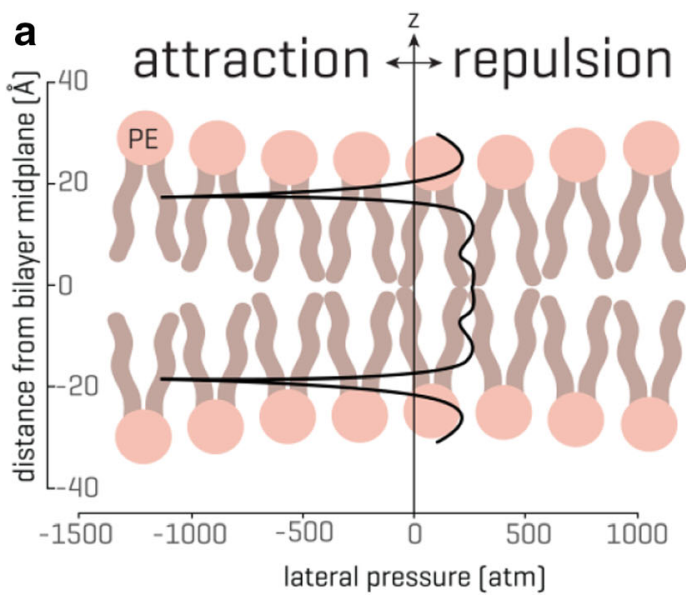

Fig. 3 Transbilayer pressure profile. The transbilayer pressure profile is largely inhomogeneous across the bilayer thickness, which originates from the amphipathic nature of the lipid molecules and the presence of water. (A) An idealized symmetrical lipid bilayer. The transbilayer pressure profile shows characteristic negative peaks at the water-lipid interface $(\sim 1000 \mathrm{~atm})$ and repulsive positive peaks $(\sim 300 \mathrm{~atm})$ in the

Transbilayer pressure profile (Fig. 3A) refers to a strong anisotropic internal stress in the lipid bilayer resulting from the amphipathic nature of the membrane lipids, which drives the bilayer self-assembly in aqueous environment by minimizing the exposure of the phospholipid aliphatic chains to water (Cantor 1999). This is because exposure of the hydrophobic lipid tails to water is energetically expensive and thus unfavorable. The hydrophilic lipid head groups, which are either polar or charged, repel each other and are pulled together due to hydrophobicity of the phospholipid tails to prevent water molecules from entering the lipid bilayer (Fig. 3A). This results in a strong attraction of phospholipid molecules at the water-lipid interface corresponding to negative pressure peaks of up to $1000 \mathrm{~atm}$. Towards the middle of the lipid bilayer, there is an increase in mobility of the lipid tails, which depends on the saturation of the lipids forming the bilayer. For mono-unsaturated phospholipid bilayers, this results in the pressure inside the bilayer of about $300 \mathrm{~atm}$ (Gullingsrud and Schulten 2004; Ridone et al. 2018). These stress/ pressure distributions within the bilayer have been mostly estimated using a variety of computational approaches. Recently, the transbilayer pressure profile of lipid bilayers made of mono- and poly-unsaturated phospholipids was experimentally determined using NMR spectroscopy, which largely confirmed the results from the computational studies. Concomitantly with patch clamp experiments, the NMR results demonstrated that changes in transbilayer pressure profile were directly related to modulation of MS channel gating (Ridone et al. 2018) in agreement with previous findings showing that the stress heterogeneity along the bilayer thickness alters the conformation of membrane proteins (Cantor 1999). Reciprocally, membrane proteins, including MS channels, can redistribute the transbilayer pressure profile causing a noticeable asymmetry in the pressure profile, which is characterized by a reduction of the pressure peaks in the tails and significant asymmetry between the peaks at the lipid solvent interface (Fig. 3B) (Cantor 1999; Lundbaek et al. 2010). Thus, when an MS channel becomes displaced in the bilayer by stretching the membrane, it is likely that the change in the bilayer pressure profile asymmetry can lead to the channel opening, as recently demonstrated for the TREK-2 ion channel (Clausen et al. 2017). In addition, the pressure profile is highly susceptible to physical and chemical stimuli (Cantor 1999), which seems to explain the effect of the insertion of amphipathic compounds into a single leaflet of the lipid bilayer, including conical lipids such as lysophosphatidylcholine (LPC) or phosphatidic acid (PA), on the activity of various MS channels, including Piezo1 (Syeda et al. 2016; Perozo et al. 2002; Maingret et al. 2000; Bavi et al. 2017a, b). Together, both the movement of an MS channel caused by membrane stretching and insertion of amphipaths into one leaflet of the lipid bilayer can activate MS channels via changes of the transbilayer pressure profile asymmetry, which thus strongly suggests that the FFL paradigm of MS channel gating presents the general evolutionary conserved physicochemical principle underlying the MS channel-mediated mechanosensory transduction in cells of all living organisms.

\section{Piezo1 adventures with lipids}

Currently, little is known about Piezo1 plasma membrane localization and organization. However, what is known is that Piezol ion channels are inherently mechanosensitive and therefore, their interactions with membrane lipids are essential for their function (Cox et al. 2016a; Syeda et al. 2016). Indeed, 
cholesterol enrichment or depletion by methyl- $\beta$-cyclodextrin (MBCD) and disruption of membrane cholesterol organization by dynasore have recently been shown to affect Piezol response to mechanical force (Ridone et al. 2019). Electrophysiological recordings in the cell-attached configuration revealed that $\mathrm{MBCD}$ caused a rightward shift in the Piezo1 pressure-response curve, increased channel latency in response to mechanical stimuli, and markedly slowed channel inactivation (Fig. 4). The same effects were seen in native Piezo1 in N2A cells.

STORM super-resolution imaging revealed that at the nanoscale, Piezo1 channels in the membrane associate as clusters sensitive to membrane manipulation. Both cluster distribution and diffusion rates were affected by treatment with $5 \mathrm{mM}$ MBCD. Supplementation of poly-unsaturated fatty acids appeared also to sensitize the Piezol response to applied pressure. Consequently, these results indicate that Piezo1 function is strongly dependent on the membrane mechanical properties and lateral organization of membrane cholesterol domains, referred to also as "lipid rafts" (Nicolson 2014),

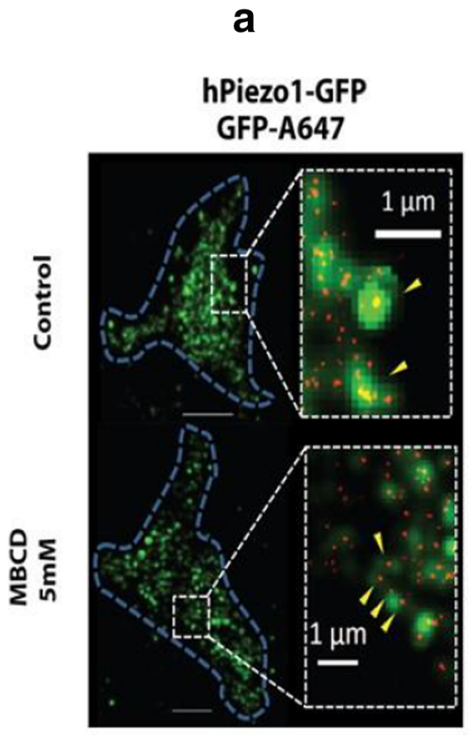

\section{Cluster Decomposition}
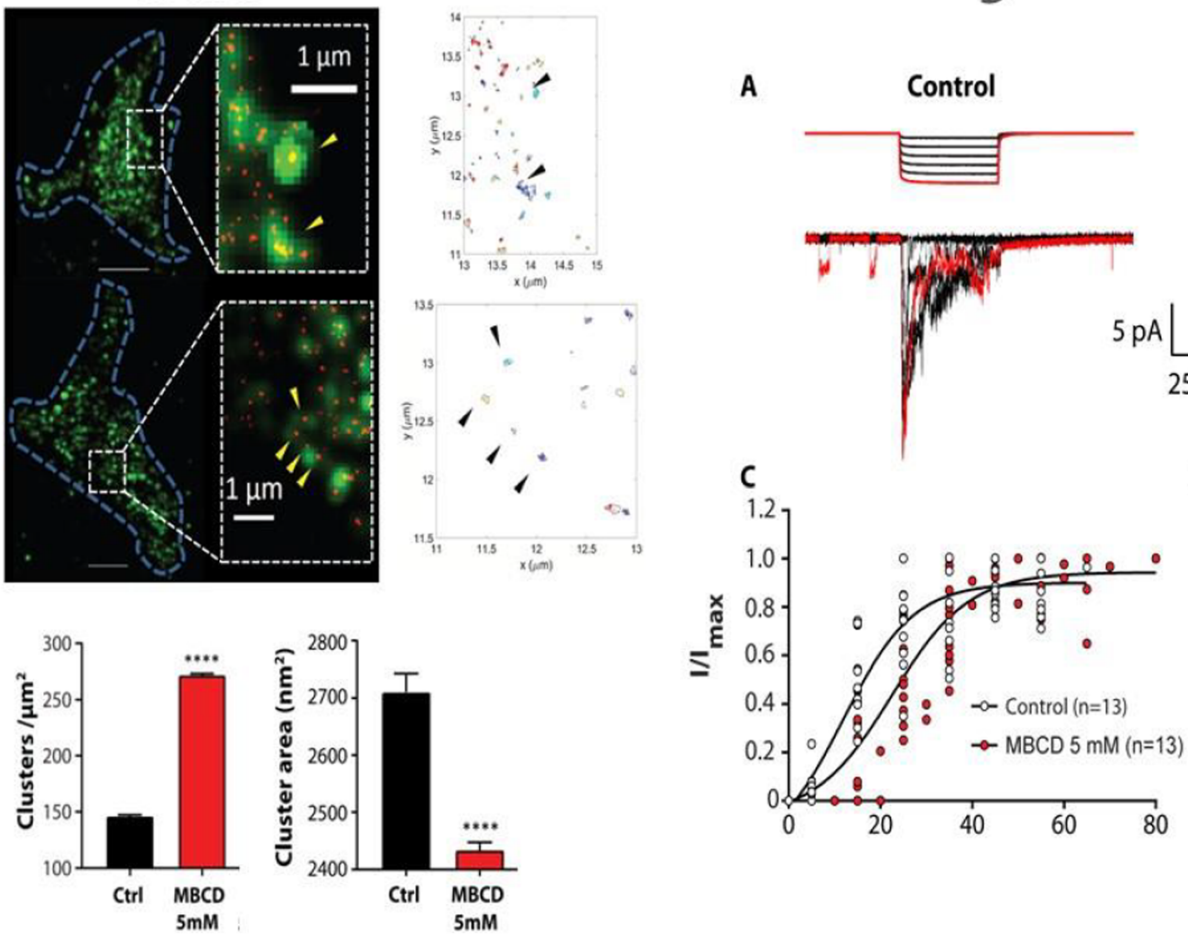

Fig. 4 Cholesterol effect on Piezo1 clustering and channel kinetics. (A) Super-resolution microscopy shows the channels clustering together at the nanoscale (top). These clusters are dependent on the cholesterol content of the membrane (bottom). (B) Leaching cholesterol from the membrane changes sensitivity and gating kinetics. Under pressure application in a membrane patch (right control) the current decays as the channel inactivates. This behavior is lost if cholesterol is removed (top).

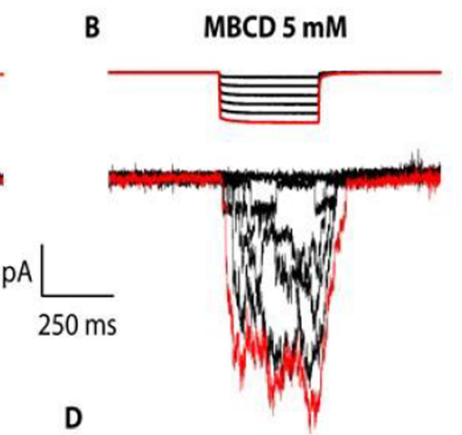

D

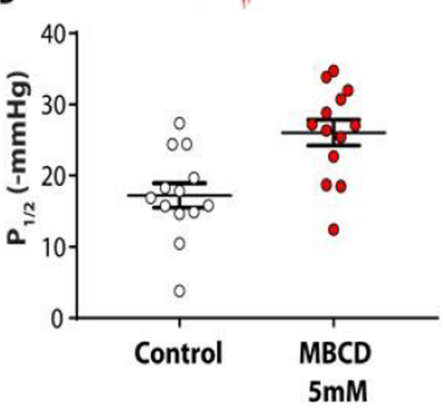

which coordinate the concerted channel activity. Lipid rafts are membrane microdomains composed of combinations of cholesterol, glycosphingolipids, and protein receptors (Nicolson 2014). Compared with the surrounding lipid bilayer, they are more ordered and tightly packed. Interestingly, cholesterol-enriched platforms such as lipid rafts are universally present in cellular force foci, which can be defined as points of force reception characteristic of specialized forms of mechanotransduction, including hearing and touch, as well as of more general cadherin foci and integrin foci (Ingber 2006). Lipid rafts thus could provide a hybrid mechanism between the FFL and FFF mechanisms of gating Piezol and other inherently mechanosensitive channels by mechanical force. Therefore, the "either-or-distinction" between the FFL and FFF paradigm seems superfluous.

In addition to cholesterol, phosphoinositides (e.g., PIP2) have been shown to be functionally relevant as well as closely related to mechanopathologies resulting from malfunction of Piezo1. During our studies of Piezol interactions with the surrounding lipid environment, we also found that not only

\section{b}

\section{Endogenous P1 expression}

Boltzmann distribution function showing dependence of Piezo 1 currents on negative pressure (suction) applied to the cell-attached membrane patch of HEK293 cells. Piezo1 channels in cells treated with $5 \mathrm{mM}$ MBCD exhibit reduced mechanosensitivity as indicated by a shallowed slope of the Boltzmann function (bottom left). The half activation pressure of Piezol channels increased significantly in membrane patches treated with MBCD (bottom right) (modified from Ridone et al. (2019)) 
PIP2 but also PIP1 and PIP3 were highly enriched around the protein (unpublished results). A binding site consisting of four lysines, K2166-K2169, in the human form of Piezo1 was identified around the channel pore domains. Notably, the four lysines are highly conserved in Piezol channel homologs. The removal of the four lysines in a deletion $\Delta 4 \mathrm{~K}$ mutant was reported to cause xerocytosis, a familial anemia characterized by a dehydrated form of red blood cells (Albuisson et al. 2013). Furthermore, in patch clamp experiments, this deletion mutation was shown to reduce channel inactivation significantly (unpublished results).

\section{Role of the cytoskeleton and extracellular matrix in Piezo1 channel gating}

Together with MS channels, cytoskeleton (CSK) is another firmly established cellular mechanosensor ${ }^{54}$.

Though similar to the bacterial MscL and MscS channels, Piezo1 also functions in reconstituted planar bilayers (Jaggers et al. 2019; Syeda et al. 2016), and critical role of lipids for the Piezo1 channel activity has clearly been established (Cox et al. 2016b). Recent experimental work conducted in our laboratory demonstrated how purified Piezol could be activated by applying pipette suction in patch clamp experiments on reconstituted proteoliposomes (Fig. 5) (unpublished results) similarly to MscL and MscS (Nomura et al. 2012).

This in no way precludes a role for the cytoskeleton in Piezo1 function (Gottlieb and Sachs 2012; Poole et al. 2014) because membrane forces are largely determined by the local arrangement of the cytoskeleton and extracellular matrix. Integrins, for example, form adhesions on the surface of mammalian cell membranes allowing mechanical stimuli to be focused on different CSK components, which directly or indirectly transmit these stimuli to membrane proteins, including ion channels (Wang et al. 1993). To fulfill this role, CSK is organized as "tensegrity" structures pre-stressing the cells to keep the cell shape stabilized by the network of opposing tension and compression components (Ingber 2006). As recently demonstrated for Piezol channels, which are gated according to the FFL paradigm (Syeda et al. 2016), the important function of the CSK and extracellular matrix (ECM) is therefore to mechanically protect the lipid bilayer by absorbing mechanical stresses (Cox et al. 2016a) and possibly also to alter the forces by modifying the time dependence of MS channel desensitization (Hamill and Martinac 2001). Furthermore, it has also been shown that the presence of some CSK proteins, such as STOML3 and tropomyosin 4.2 (Tpm4.2), apparently prestresses the cell membrane, which results in increased mechanosensitivity of Piezol to membrane tension (Cox et al. 2018). Alternatively, the presence of filamin A was shown to reduce the mechanosensitivity of the channel.
Moreover, an atomic force microscopy (AFM) study reported that mechanical stimulation of Piezo1 was dependent on the channel connections/interactions with ECM proteins (Gaub and Muller 2017). Piezol channels were relatively insensitive to mechanical stimuli pushing the cell membrane, whereas forces pulling the membrane could activate the channel effectively. The protein that was found to sensitize Piezo1 to pulling forces was identified as collagen IV, which is a component of the basal lamina forming a cohesive network and mechanical connection between cells. Consequently, not only the direct interaction between Piezol and ECM but also directionality of mechanical force can modulate Piezo1 sensitivity to mechanical stimuli.

\section{Piezo1 channel function in the (patho) physiology of mechanotransduction}

The importance of the malfunctioning MS ion channels as contributors to pathology of a variety of hereditary diseases of previously unknown etiology has increasingly caught the attention of medical practitioners. This includes a variety of mechanochannelopathies where point mutations in an MS channel can be traced as the causative entity.

Despite their relatively recent discovery Piezo, ion channels have already been closely linked to many processes defining mechanotransduction processes in mammalian cells. Both Piezo1 and Piezo2 are widely expressed in the tissues of hollow organs such as the stomach, lungs, bladder, intestines, and endothelial cells lining the lumen of blood vessels (Martinac and Cox 2017). The central role they seem to play in mammalian organisms is underlined by the fact that global knockouts of Piezo1 in mice result in embryonic lethality ( $\mathrm{Li}$ et al. 2014). Furthermore, gain-of-function (GOF) mutations in human Piezo1 cause hereditary xerocytosis (also known as dehydrated stomatocytosis), a familial anemia (Bae et al. 2013; Gottlieb and Sachs 2012), whereas loss-of-function (LOF) mutations cause generalized lymphatic dysplasia characterized by varying degrees of anemia (Fotiou et al. 2015; Lukacs et al. 2015). Both channelopathies suggest a central role that Piezol plays in erythrocyte volume control. As the research on MS ion channels continues progressing and the new knowledge of MS channel structure and function continues growing, new MS channel proteins may become discovered and new links between malfunctioning MS channels and related mechanopathophysiology can be found. Thus, our awareness of diseases linked to malfunctioning MS channels will increase and will make MS channels attractive targets for the development of new drugs to treat a variety of mechanochannelopathies in the future.

Studies on the effects of lipids such as cholesterol on PIEZO1 activity (Romero et al. 2019; Ridone et al. 2019) suggest that signal compartmentalization from specific membrane 
a hPIEZO1 channels in PE:PC:PG:Cholesterol liposomes
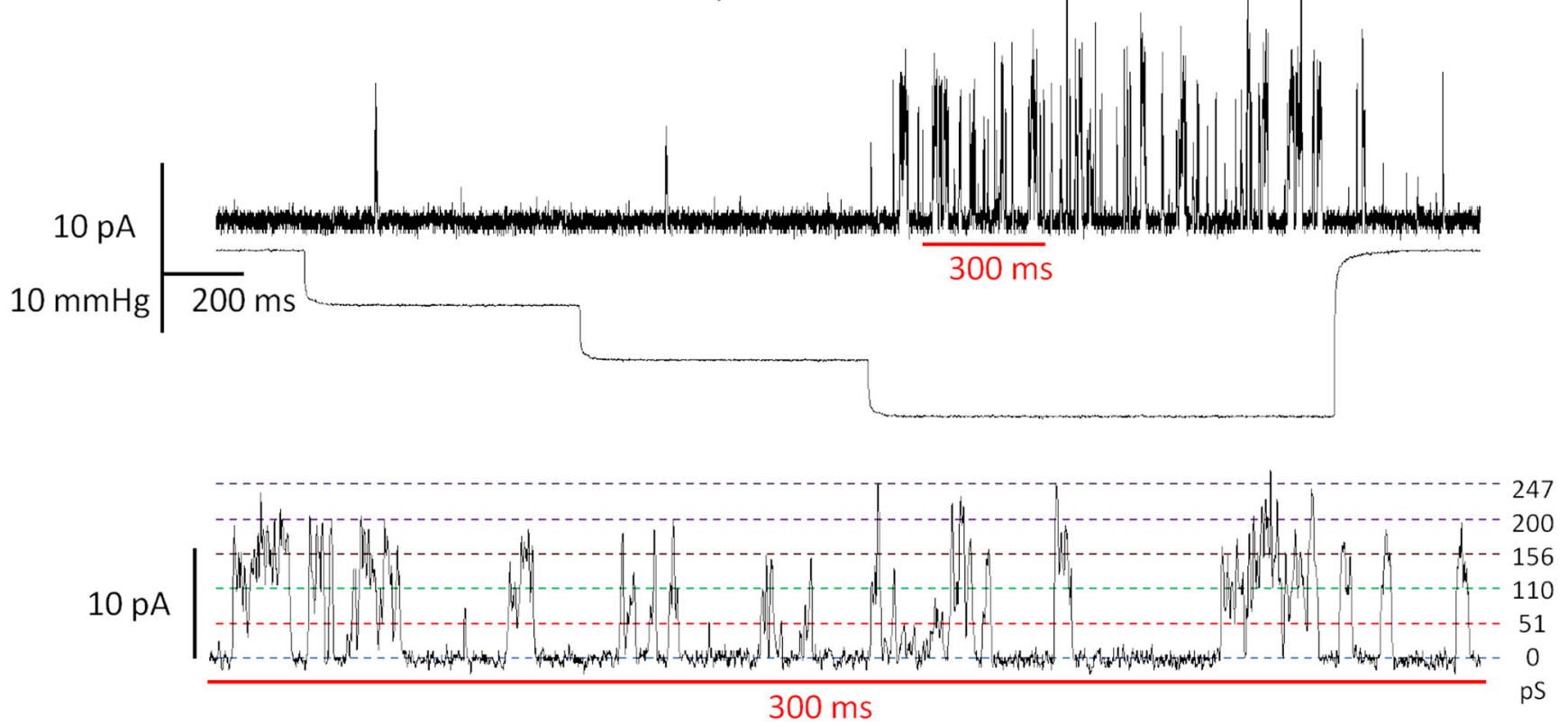

b Single hPIEZO1 channel currents

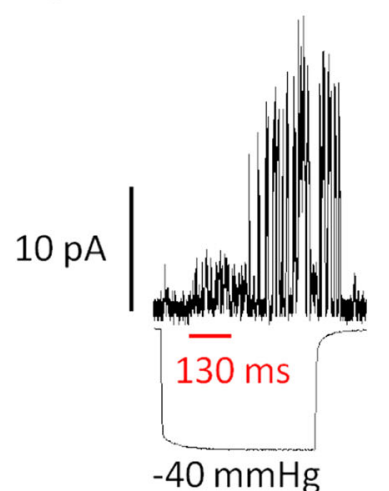

Fig. 5 Mechanical activation of purified human Piezo1 (hPiezo1) reconstituted in artificial liposomes made of PE:PC:PG:Cholesterol. (A) Representative inside-out patch clamp recording of hPIEZO1 at $+65 \mathrm{mV}$ pipette potential. Inward currents produced in response to $10 \mathrm{mmHg}$ incremental steps in suction. Bottom: inset shows the concerted activity of up to $5 \mathrm{hPIEZO1}$ channels and the observed conductance values (pS) are indicated on the right. (B) Mechanical activation of single PIEZO1 channel recorded in artificial liposomes and summarized in a representative

regions might explain how the conduction of such a ubiquitous ion such as $\mathrm{Ca}^{2+}$ could trigger very specific mechanosensitive responses. All regulatory modes described above (CSK, ECM, lipid rafts, protein-protein interactions, and channel clustering) ultimately fine-tune the sensitivity, magnitude, and duration of Piezo1 currents. Compartmentalization of mechanosensitive signaling facilitates an efficient and specific transduction process to achieve localized subcellular remodeling. Tensile forces are instantaneously transmitted across large distances and the remodeling action of the actin-severing machinery can impact mechanosensitivity and force-dependent signaling at locations far from the point of force detection (force foci) (Burridge and
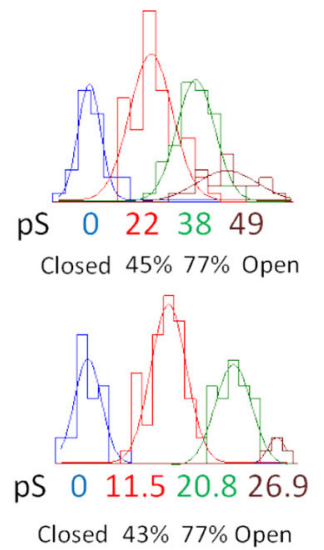

histogram of conductance values on the right-hand side. Bottom: single hPIEZO1 activity in HEK293T cells transfected with the same construct used for protein purification and representative histogram of single channel events (liposome recording solution: $200 \mathrm{mM} \mathrm{KCl}, 40 \mathrm{mM} \mathrm{MgCl}$, $5 \mathrm{mM}$ HEPES, pH 7.4. HEK293 cell recoding solution: $140 \mathrm{mM} \mathrm{NaCl}$, $3 \mathrm{mM} \mathrm{KCl}, 1 \mathrm{mM} \mathrm{MgCl} 2,1 \mathrm{mM} \mathrm{CaCl} 2,10 \mathrm{mM}$ glucose, $10 \mathrm{mM}$ HEPES, $\mathrm{pH}$ 7.4) (unpublished results)

Guilluy 2016). The Piezo1 channel is in fact ideally placed in the cellular context to respond to both "Outside-In" (external stimuli) and "Inside-Out" (cell-generated) signaling (Kwong et al. 2003). This results in not only biochemical feedback loops (e.g., filamin A (Retailleau et al. 2015) and myosin II (Ellefsen et al. 2018)) but also genetic feedback loops that drive the overexpression of Piezo1 channels, as described in multiple cell types from animal models and in vitro (Satoh et al. 2006; Liu et al. 2018; Jones et al. 2018; Etem et al. 2018; VelascoEstevez et al. 2018; Jin et al. 2015; Liang et al. 2017; Michishita et al. 2016). While biochemical feedbacks might impact the rearrangement events on the short time scales, the 
genetic feedbacks represent critically relevant mediators of pathology and cancer since they have a long-term impact on the activity of the many oncogenic pathways linked to Piezol (e.g., HIPPO, MAPK, ERK).

\section{Pharmacology of Piezo1 mechanosensitive channels}

At present, there are only a few agents and compounds generally used for applications in MS channel research. They include the lanthanides $\mathrm{Gd}^{3+}$ and $\mathrm{La}^{3+}$, the aminoglycoside antibiotics such as streptomycin and gentamicin, and the GsMTx-4 peptide isolated from the toxin of the spider Grammostola spatulata, which are known to block most of the known MS channels (Martinac and Cox 2017; Hamill and McBride 1996). Another group of compounds, such as amphipaths chlorpromazine and LPC, have been shown to activate both prokaryotic and eukaryotic MS channels (Martinac and Cox 2017; Hamill and McBride 1996). In addition to these "typical" MS channel blockers and activators, there are more specific compounds affecting only a certain type of MS channels. For example, ruthenium red and Yoda1 block and activate Piezo1, respectively (Coste et al. 2012; Syeda et al. 2015). More recently, an analog of Yoda1, named Dooku1, was synthetized and shown to reversibly antagonize Yoda1-induced activation of Piezo1 by competing for a specific channel binding site (Evans et al. 2018). A comprehensive and up-to-date list of blocking and activating agents of various types of MS channels can be found in Martinac and Cox (2017).

\section{Conclusions}

In this review, we attempted to illustrate generally as well as specifically on the example of Piezo1 channels what makes MS channels unique and why we believe it is important to study them and to understand their biophysical gating principles. These channels are the force-sensing molecules providing information about the outside world through direct contact cells have with their surroundings. Sometimes, this information may not be exactly transduced into a proper intracellular signaling due to aberrant channel function, as it is apparently the case in hereditary mechanopathologies we briefly reported here. Our knowledge of the structure and function of MS channels comes to the rescue by helping us to understand the correct etiology of these pathologies. This emphasizes the need for more knowledge and better understanding of the MS channel diversity and their equally diverse structure and function, which can only be achieved through continuous discoveries and painstaking research. Despite the progress that has been made over the past 30 odd years in this field, there are still many outstanding questions. Thus, we look forward to future developments that may open new avenues for the treatment of a whole host of mechanochannelopathies, which should enrich our knowledge and improve human health conditions.

\section{Glossary}

Amphipaths

A term describing a chemical compound possessing both hydrophilic and lipophilic properties.

Archaea A group of single-celled microorganisms, which like bacteria have no cell nucleus or any other organelles within their cells.

GPCRs $\mathrm{G}$ protein-coupled receptors are cell surface receptors activated by different types of stimuli, including light and binding of peptides, proteins, and lipids. They form the largest and highly diverse group of membrane receptors in eukaryotic organisms.

GsMTx-4 One of the peptide toxins isolated from the venom of the Chilean rose tarantula spider Grammostola spatulata that is known to block mechanosensitive ion channels.

HEK293 cells Human embryonic kidney 293 cells frequently used in cell biology and biotechnology for transfection and production of viral vectors, vaccines, and recombinant proteins.

MscL

MscS

N2A cells

OSCA

Bacterial mechanosensitive channel of large conductance.

Bacterial mechanosensitive channel of small conductance.

Neuro2a cells are a fast-growing mouse neuroblastoma cell line.

A new class of mechanosensitive calcium-permeable ion channels in plants that are activated by high osmotic shock.

PE, PC, and PG Abbreviations for major phospholipid constituents of biological membranes: phosphatidylethanolamine, phosphatidylcholine, and phosphatidylglycerol, respectively.

STOML3 Stomatin-like protein-3, which controls cell membrane mechanics by binding cholesterol.

STORM Stochastic optical reconstruction microscopy is a super-resolution imaging technique utilizing sequential 
activation and time-resolved localization of photo-switchable fluorophores to create high-resolution images.

TREK-1, TREK-2, Mechanosensitive members of the and TRAAK

two-pore-domain background potassium (K2P) channel protein family, which includes functionally distinct subgroups: TWIK, THIK, TASK TALK, TREK, and TRESK.

Open Access This article is distributed under the terms of the Creative Commons Attribution 4.0 International License (http:// creativecommons.org/licenses/by/4.0/), which permits unrestricted use, distribution, and reproduction in any medium, provided you give appropriate credit to the original author(s) and the source, provide a link to the Creative Commons license, and indicate if changes were made.

\section{References}

Albuisson J, Murthy SE, Bandell M, Coste B, Louis-Dit-Picard H, Mathur J, Feneant-Thibault M, Tertian G, De Jaureguiberry JP, Syfuss PY, Cahalan S, Garcon L, Toutain F, Simon Rohrlich P, Delaunay J, Picard V, Jeunemaitre X, Patapoutian A (2013) Dehydrated hereditary stomatocytosis linked to gain-of-function mutations in mechanically activated PIEZO1 ion channels. Nat Commun 4:1884

Bae C, Gnanasambandam R, Nicolai C, Sachs F, Gottlieb PA (2013) Xerocytosis is caused by mutations that alter the kinetics of the mechanosensitive channel PIEZO1. Proc Natl Acad Sci U S A 110:E1162-E1168

Barry RM, Gitai Z (2011) Self-assembling enzymes and the origins of the cytoskeleton. Curr Opin Microbiol 14:704-711

Bass RB, Strop P, Barclay M, Rees DC (2002) Crystal structure of Escherichia coli MscS, a voltage-modulated and mechanosensitive channel. Science 298:1582-1587

Bavi N, Nakayama Y, Bavi O, Cox CD, Qin QH, Martinac B (2014) Biophysical implications of lipid bilayer rheometry for mechanosensitive channels. Proc Natl Acad Sci U S A 111: 13864-13869

Bavi N, Cortes M, Cox CD, Rohde PR, Liu W, Deitmer JW, Bavi O, Strop P, Hill AP, Rees D, Corry B, Perozo E, Martinac B (2016) The role of MscL amphipathic $\mathrm{N}$ terminus indicates a blueprint for bilayer-mediated gating of mechanosensitive channels. Nat Commun: 7

Bavi, N., Cox, C.D., Bavi, O. and Martinac, B. Perturbation of bilayer surface tension differentially modulates mechanosensitive ion channels 61st Annual Meeting of the Biophysical Society, 2017a New Orleans. Biophys J

Bavi N, Nikolaev YA, Bavi O, Ridone P, Martinac AD, Nakayama Y, Cox CD and Martinac B. (2017b) Principles of mechanosensing at the membrane interface In: R.M EPAND, J.-M. R. (ed.) The biophysics of cell membranes. Singapore: Springer Nature

Berrier C, Pozza A, De Lacroix De Lavalette A, Chardonnet S, Mesneau A, Jaxel C, Le Maire M, Ghazi A (2013) The purified mechanosensitive channel TREK-1 is directly sensitive to membrane tension. J Biol Chem 288:27307-27314

Brohawn SG, Del Marmol J, Mackinnon R (2012) Crystal structure of the human K2P TRAAK, a lipid- and mechano-sensitive $\mathrm{K}+$ ion channel. Science 335:436-441
Brohawn SG, Campbell EB, Mackinnon R (2014a) Physical mechanism for gating and mechanosensitivity of the human TRAAK $\mathrm{K}+$ channel. Nature 516:126-130

Brohawn SG, Su Z, Mackinnon R (2014b) Mechanosensitivity is mediated directly by the lipid membrane in TRAAK and TREK1 K+ channels. Proc Natl Acad Sci U S A 111:3614-3619

Burridge K, Guilluy C (2016) Focal adhesions, stress fibers and mechanical tension. Exp Cell Res 343:14-20

Cantor RS (1999) Lipid composition and the lateral pressure profile in bilayers. Biophys J 76:2625-2639

Chalfie M (2009) Neurosensory mechanotransduction. Nat Rev Mol Cell Biol 10:44-52

Chang G, Spencer RH, Lee AT, Barclay MT, Rees DC (1998) Structure of the MscL homolog from Mycobacterium tuberculosis: a gated mechanosensitive ion channel. Science 282:2220-2226

Clausen MV, Jarerattanachat V, Carpenter EP, Sansom MSP, Tucker SJ (2017) Asymmetric mechanosensitivity in a eukaryotic ion channel. Proc Natl Acad Sci U S A 114:E8343-E8351

Coste B, Mathur J, Schmidt M, Earley TJ, Ranade S, Petrus MJ, Dubin AE, Patapoutian A (2010) Piezo1 and Piezo2 are essential components of distinct mechanically activated cation channels. Science 330:55-60

Coste B, Xiao B, Santos JS, Syeda R, Grandl J, Spencer KS, Kim SE, Schmidt M, Mathur J, Dubin AE, Montal M, Patapoutian A (2012) Piezo proteins are pore-forming subunits of mechanically activated channels. Nature 483:176-181

Cox CD, Bae C, Ziegler L, Hartley S, Nikolova-Krstevski V, Rohde PR, Ng CA, Sachs F, Gottlieb PA, Martinac B (2016a) Removal of the mechanoprotective influence of the cytoskeleton reveals PIEZO1 is gated by bilayer tension. Nat Commun 7:10366

Cox, C. D., Bavi, N. \& Martinac, B. 2016b. Origin of the force: the forcefrom-lipids principle applied to piezo channels. Current topics in membranes. Academic Press

Cox CD, Bavii N, Martinac B (2018) Cytoskeleton-associated proteins modulate the tesnion sensitivity of PIEZO1. Biophys J 114:A562

Dong YY, Pike AC, Mackenzie A, Mcclenaghan C, Aryal P, Dong L, Quigley A, Grieben M, Goubin S, Mukhopadhyay S, Ruda GF, Clausen MV, Cao L, Brennan PE, Burgess-Brown NA, Sansom MS, Tucker SJ, Carpenter EP (2015) K2P channel gating mechanisms revealed by structures of TREK-2 and a complex with Prozac. Science 347:1256-1259

Ellefsen K, Chang A, Holt JR, Nourse JL, Arulmoli J, Mekhdjian A, Abuwarda H, Tombola F, Flanagan LA, Dunn AR et al (2018) Myosin-II mediated traction forces evoke localized Piezo1 $\mathrm{Ca}^{2+}$ flickers. bioRxiv, 294611

Etem EO, Ceylan GG, Ozaydin S, Ceylan C, Ozercan I, Kuloglu T (2018) The increased expression of Piezo1 and Piezo2 ion channels in human and mouse bladder carcinoma. Adv Clin Exp Med 27:10251031

Evans EL, Cuthbertson K, Endesh N, Rode B, Blythe NM, Hyman AJ, Hall SJ, Gaunt HJ, Ludlow MJ, Foster R, Beech DJ (2018) Yoda1 analogue (Dooku1) which antagonizes Yoda1-evoked activation of Piezo1 and aortic relaxation. Br J Pharmacol 175:1744-1759

Fotiou E, Martin-Almedina S, Simpson MA, Lin S, Gordon K, Brice G, Atton G, Jeffery I, Rees DC, Mignot C, Vogt J, Homfray T, Snyder MP, Rockson SG, Jeffery S, Mortimer PS, Mansour S, Ostergaard P (2015) Novel mutations in PIEZO1 cause an autosomal recessive generalized lymphatic dysplasia with non-immune hydrops fetalis. Nat Commun 6:8085

Gaub BM, Muller DJ (2017) Mechanical stimulation of Piezo1 receptors depends on extracellular matrix proteins and directionality of force. Nano Lett 17:2064-2072

Ge J, Li W, Zhao Q, Li N, Chen M, Zhi P, Li R, Gao N, Xiao B, Yang M (2015) Architecture of the mammalian mechanosensitive Piezol channel. Nature 527:64-69 
Gillespie PG, Walker RG (2001) Molecular basis of mechanosensory transduction. Nature 413:194-202

Gottlieb PA, Sachs F (2012) Piezo1: properties of a cation selective mechanical channel. Channels (Austin) 6:214-219

Gullingsrud J, Schulten K (2004) Lipid bilyer pressure profiles and mechanosensitive channel gating. Biophys J 86(6):3496-3509

Guo YR \& Mackinnon R (2017) Structure-based membrane dome mechanism for Piezo mechanosensitivity. Elife, 6

Guo YRAMR (2017) Structure-based membrane dome mechanism for piezo mechanosensitivity. eLife 6:e33660

Hamill OP, Martinac B (2001) Molecular basis of mechanotransduction in living cells. Physiol Rev 81:685-740

Hamill OP, McBride DW Jr (1996) The pharmacology of mechanogated membrane ion channels. Pharmacol Rev 48:231-252

HonorÉ E, Martins JR, Penton D, Patel A, Demolombe S (2015) The piezo mechanosensitive ion channels: may the force be with you! Rev Physiol Biochem Pharmacol 169:25-41

Ingber DE (2006) Cellular mechanotransduction: putting all the pieces together again. FASEB J 20:811-827

Jaggers OB, Ridone P, Martinac B, Baker MAB (2019) Fluorescence microscopy of piezol in droplet hydrogel bilayers. Channels (Austin) 13:102-109

Jin Y, Li J, Wang YT, Ye R, Feng XX, Jing Z, Zhao ZH (2015) Functional role of mechanosensitive ion channel Piezo1 in human periodontal ligament cells. Angle Orthod 85:8794

Jones RC, Ter Heegde F, Jackson TR, O'brien M, Board TN, Richardson SM, Townsend PA, Lawrence KM (2018) Piezo1 expression is increased in response to non-invasive impact of mouse knee joint. Osteoarthr Cartilage 26:S113-S114

Katta S, Krieg M, Goodman MB (2015) Feeling force: physical and physiological principles enabling sensory mechanotransduction. Annu Rev Cell Dev Biol 31:347-371

Kocer A (2015) Mechanisms of mechanosensing - mechanosensitive channels, function and re-engineering. Curr Opin Chem Biol 29: $120-127$

Kung C (2005) A possible unifying principle for mechanosensation. Nature 436:647-654

Kwong L, Wozniak MA, Collins AS, Wilson SD, Keely PJ (2003) R-Ras promotes focal adhesion formation through focal kinase and p130(Cas) by a novel mechanism that differs from integrins. Mol Cell Biol 23:933-949

Lehtonen JY, Kinnunen PK (1995) Phospholipase A2 as a mechanosensor. Biophys J 68:1888-1894

Li J, Hou B, Tumova S, Muraki K, Bruns A, Ludlow MJ, Sedo A, Hyman AJ, McKeown L, Young RS, Yuldasheva NY, Majeed Y, Wilson LA, Rode B, Bailey MA, Kim HR, Fu Z, Carter DA, Bilton J, Imrie H, Ajuh P, Dear TN, Cubbon RM, Kearney MT, Prasad RK, Evans PC, Ainscough JF, Beech DJ (2014) Piezo1 integration of vascular architecture with physiological force. Nature 515:279-282

Liang JL, Huang BS, Yuan GY, Chen Y, Liang FS, Zeng HY, Zheng SX, Cao L, Geng DF, And Zhou, S.X. (2017) Stretch-activated channel Piezol is up-regulated in failure heart and cardiomyocyte stimulated by AngII. Am J Transl Res 9:2945-2955

Liu Q, Sun BS, Zhao J, Wang QQ, An F, Hu XY, Yang ZX, Xu J, Tan MJ, Li LK (2018) Increased Piezo1 channel activity in interstitial Cajallike cells induces bladder hyperactivity by functionally interacting with NCX1 in rats with cyclophosphamide-induced cystitis. Exp Mol Med 50

Lukacs V, Mathur J, Mao R, Bayrak-Toydemir P, Procter M, Cahalan SM, Kim HJ, Bandell M, Longo N, Day RW, Stevenson DA, Patapoutian A, Krock BL (2015) Impaired Piezol function in patients with a novel autosomal recessive congenital lymphatic dysplasia. Nat Commun 6:8329
Lundbaek JA, Collingwood SA, Ingolfsson HI, Kapoor R, Andersen OS (2010) Lipid bilayer regulation of membrane protein function: gramicidin channels as molecular force probes. J R Soc Interface 7:373395

Maingret F, Patel AJ, Lesage F, Lazdunski M, Honore E (2000) Lysophospholipids open the two-pore domain mechano-gated $\mathrm{K}(+$ ) channels TREK-1 and TRAAK. J Biol Chem 275:10128-10133

Martinac B (2004) Mechanosensitive ion channels: molecules of mechanotransduction. J Cell Sci 117:2449-2460

Martinac B (2011) Bacterial mechanosensitive channels as a paradigm for mechanosensory transduction. Cell Physiology and Biochemistry 28:1051-1060

Martinac B (2013) The ion channels to cytoskeleton connection as potential mechanism of mechanosensitivity. Biochim Biophys Acta

Martinac B, Cox CD (2017) Mechanosensory transduction: focus on ion channels. Elsevier, Comprehensive Biophysics

Martinac B, Adler J, Kung C (1990) Mechanosensitive ion channels of E. coli activated by amphipaths. Nature 348:261-263

Michishita M, Yano K, Tomita KI, Matsuzaki O, And Kasahara, K.I (2016) Piezo1 expression increases in rat bladder after partial bladder outlet obstruction. Life Sci 166:1-7

Murthy SE, Dubin AE, Whitwam T, Jojoa-Cruz S, Cahalan SM, Mousavi SAR, Ward AB, Patapoutian A (2018) Osca/Tmem63 are an evolutionarily conserved family of mechanically activated ion channels. Elife 7

Nicolson GL (2014) The Fluid-Mosaic Model of membrane structure: still relevant to understanding the structure, function and dynamics of biological membranes after more than 40 years. Biochim Biophys Acta 1838:1451-1466

Nomura T, Cranfield CG, Deplazes E, Owen DM, Macmillan A, Battle AR, Constantine M, Sokabe M, Martinac B (2012) Differential effects of lipids and lyso-lipids on the mechanosensitivity of the mechanosensitive channels MscL and MscS. Proc Natl Acad Sci U S A 109:8770-8775

Perozo E, Kloda A, Cortes DM, Martinac B (2002) Physical principles underlying the transduction of bilayer deformation forces during mechanosensitive channel gating. Nat Struct Biol 9:696-703

Poole K, Herget R, Lapatsina L, Ngo HD, Lewin GR (2014) Tuning Piezo ion channels to detect molecular-scale movements relevant for fine touch. Nat Commun 5:3520

Ranade SS, Syeda R, Patapoutian A (2015) Mechanically activated ion channels. Neuron 87:1162-1179

Retailleau K, Duprat F, Arhatte M, Ranade SS, Peyronnet R, Martins JR, Jodar M, Moro C, Offermanns S, Feng Y et al (2015) Piezo1 in smooth muscle cells is involved in hypertension-dependent arterial remodeling. Cell Rep 13:1161-1171

Ridone P, Grage SL, Patkunarajah A, Battle AR, Ulrich, A.S. AND Martinac, B. (2018) "Force-from-lipids" gating of mechanosensitive channels modulated by PUFAs. J Mech Behav Biomed Mat 79:158167

Ridone, P., Pandzic, E., Vassalli, M., Cox, C.D., Macmillan, A., Gottlieb, P.A. and Martinac, B. (2019) Disruption of membrane cholesterol organization impairs the concerted activity of PIEZO1 channel clusters. BioRxiv

Romero LO, Massey AE, Mata-Daboin AD, Sierra-Valdez FJ, Chauhan SC, Cordero-Morales JF, Vasquez V (2019) Dietary fatty acids finetune Piezo1 mechanical response. Nat Commun 10

Sachs F (2010) Stretch-activated ion channels: what are they? Physiology 25:50-56

Saotome K, Murthy SE, Kefauver JM, Whitwam T, Patapoutian A, Ward AB (2018) Structure of the mechanically activated ion channel Piezo1. Nature 554:481-486 
Satoh K, Hata M, Takahara S, Tsuzaki H, Yokota H, Akatsu H, Yamamoto T, Kosaka, K. AND Yamada. T. (2006) A novel membrane protein, encoded by the gene covering KIAA0233, is transcriptionally induced in senile plaque-associated astrocytes. Brain Res 1108:19-27

Storch U, Mederos Y, Schnitzler M, Gudermann T (2012) G proteinmediated stretch reception. Am J Physiol Heart Circ Physiol 302: H1241-H1249

Syeda R, Xu J, Dubin AE, Coste B, Mathur J, Huynh T, Matzen J, Lao J, Tully DC, Engels IH, Petrassi HM, Schumacher AM, Montal M, Bandell M, Patapoutian A (2015) Chemical activation of the mechanotransduction channel Piezo1. Elife 4

Syeda R, Florendo MN, Cox CD, Kefauver JM, Santos JS, Martinac B, Patapoutian A (2016) Piezol channels are inherently mechanosensitive. Cell Rep 17:1739-1746

Teng J, Loukin S, Anishkin A, Kung C (2015) The force-from-lipid (FFL) principle of mechanosensitivity, at large and in elements. Pflugers Arch
Velasco-Estevez M, Mampay M, Boutin N, Chaney A, Warn P, Sharp A, Burgess E, Moeendarbary E, Dev KK, Sheridan GK0 (2018) Infection augments expression of mechanosensing Piezol channels in amyloid plaque-reactive astrocytes. Front Aging Neurosci 1

Wang N, Butler JP, Ingber DE (1993) Mechanotransduction across the cell surface and through the cytoskeleton. Science 260:1124-1127

Wang L, Zhou H, Zhang M, Liu W, Deng T, Zhao Q, Li Y, Lei J, Li X, Xiao B (2019) Structure and mechanogating of the mammalian tactile channel PIEZO2. Nature

Zhao Q, Zhou H, Chi S, Wang Y, Wang J, Geng J, Wu K, Liu W, Zhang T, Dong MQ, Wang J, Li X, Xiao B (2018) Structure and mechanogating mechanism of the Piezol channel. Nature 554:487-492

Publisher's note Springer Nature remains neutral with regard to jurisdictional claims in published maps and institutional affiliations. 